\title{
artigo
}

Ribeiro, C.L.; Souza, J.F.; Pereira, L.P.; Brasil. R.F.G.; Santos, V.F.; Vieira, L.J.E.S.;

Atuação do enfermeiro na preservação de vestígios forenses nos casos de violência sexual: revisão integrativa

\section{Atuação do enfermeiro na preservação de vestígios forenses nos casos de violência sexual: revisão integrativa}

\author{
Performance of nurses in the preservation of forensic traces in cases of sexual violence: integrative review \\ Desempeño de enfermeras en la conservación de huellas forenses en casos de violencia sexual: revisión integrativa
}

\begin{abstract}
RESUMO
Objetivo: Descrever como atua o enfermeiro na preservação de vestígios forenses em casos de violência sexual. Métodos: revisão integrativa realizada por meio das bases de dados Literatura Latino-Americana e do Caribe em Ciências da Saúde (LILACS), Base de Dados em Enfermagem (BDENF) e Scientific Electronic e Library Online (Scielo). O período da coleta aconteceu no mês de Setembro de 2020. Resultados: Na presente revisão integrativa, analisou-se 06 artigos que atenderam aos critérios de inclusão previamente estabelecidos e, a seguir, apresentou-se um panorama geral dos artigos avaliados. A escassez de conhecimento científico referente à violência e enfermagem forense levam aos profissionais de saúde encontrarem dificuldades na utilização de técnicas específicas para a preservação dos indícios. Conclusão: o enfermeiro forense possui uma gama de responsabilidade perante os sobreviventes de violência sexual, desde a abordagem até o manuseio adequado das provas.
\end{abstract}

DESCRITORES: Cuidados de Enfermagem, Delitos Sexuais, Enfermagem Forense, Saúde da Mulher, Serviços de Saúde da Mulher.

\section{ABSTRACT}

Objective: To describe how nurses work to preserve forensic remains in cases of sexual violence. Methods: integrative review carried out using the Latin American and Caribbean Literature in Health Sciences databases (LILACS), Nursing Database (BDENF) and Scientific Electronic and Library Online (Scielo). The collection period took place in September 2020. Results: In this systematic review, 06 articles were analyzed that met the inclusion criteria previously established and, next, an overview of the evaluated articles was presented. The scarcity of scientific knowledge regarding violence and forensic nursing leads health professionals to encounter difficulties in the use of specific techniques for the preservation of evidence. Conclusion: the forensic nurse has a range of responsibility towards survivors of sexual violence, from the approach to the proper handling of evidence. DESCRIPTORS: Nursing Care, Sex Offenses, Forensic Nursing, Women's Health, Women's Health Services.

\section{RESUMEN}

Objetivo: Describir cómo trabajan las enfermeras en la preservación de rastros forenses en casos de violencia sexual. Métodos: revisión integradora realizada utilizando las bases de datos de Literatura Latinoamericana y Caribeña en Ciencias de la Salud (LILACS), Base de Datos de Enfermería (BDENF) y Bases de Datos Científico Electrónico y de Biblioteca en Línea (Scielo). El período de recolección tuvo lugar en septiembre de 2020. Resultados: En esta revisión integradora se analizaron 06 artículos que cumplieron con los criterios de inclusión avanzada y, a continuación, se presenta un resumen de los artículos obtenidos. La falta de conocimiento científico sobre la violencia y la enfermería forense lleva a los profesionales de la salud a encontrar dificultades en el uso de técnicas específicas para preservar la evidencia. Conclusión: el enfermero forense tiene un rango de responsabilidad hacia las sobrevivientes de violencia sexual, desde el abordaje hasta el adecuado manejo de las pruebas

DESCRIPTORES: Atención de Enfermería, Delitos Sexuales, Enfermería Forense, Salud de la Mujer, Servicios de Salud de la Mujer.

RECEBIDO EM: 07/04/2021 APROVADO EM: 16/04/2021

\section{Camila Lima Ribeiro}

Universidade de Fortaleza. Mestranda em Saúde Coletiva- PPGSC/UNIFOR. Especialista em Ginecologia e Obstetrícia - UNIQ; Especializanda em Enfermagem Forense - Unyleya.

ORCID: 0000-0002-1599-8454 


\section{Joice Fabricio de Souza}

Universidade de Fortaleza - Mestranda em Saúde Coletiva - PPGSC/UNIFOR. Bolsista pela Fundação Cearense de Amparo ao Desenvolvimento Científico e Tecnológico- FUNCAP. Integrante do Laboratório de Psicopatologia e Clínica Humanista Fenomenológica- APHETO, do Núcleo de Fenomenologia e Saúde - NUFES e do Grupo de Pesquisa Clínica, Cuidado e Gestão em Saúde - GPCLIN/URCA.

ORCID: 0000-0002-3165-1135

\section{Lívia de Paulo Pereira}

Maternidade-Escola Assis Chateaubriand - MEAC. Enfermeira. Doutora em Biotecnologia. ORCID :0000-0001-8626-2674

\section{Raquel Ferreira Gomes Brasil}

Maternidade-Escola Assis Chateaubriand - MEAC. Enfermeira. Especialista em Enfermagem do trabalho. Mestra em Enfermagem. ORCID: 0000-0002-8600-4410

\section{Vanessa da Frota Santos}

Maternidade-Escola Assis Chateaubriand - MEAC. Enfermeira. Doutora em Enfermagem. ORCID: 0000-0002-1198-6560

\section{Luiza Jane Eyre de Souza Vieira}

Universidade de Fortaleza. Mestre em Enfermagem Comunitária pela Universidade Federal do Ceará (1997), doutorado em Enfermagem pela Universidade Federal do Ceará (2001) e Pós-Doutorado em Saúde Coletiva pelo Programa de Pós-Graduação em Saúde Coletiva do Instituto de Saúde Coletiva-ISC, Universidade Federal da Bahia (2017). Professora titular da Universidade de Fortaleza, vinculada ao curso de Enfermagem e ao Programa de Pós-Graduação em Saúde Coletiva (PPGSC). ORCID: 0000-0002-5220-027X

\section{INTRODUÇÃO}

A IAFN (International Association of Forensic Nursing), refere que a enfermagem forense é uma especialidade definida como a aplicação da ciência da enfermagem a população e à justiça. Seu principal objetivo é oferecer suporte as investigações em vítimas dos mais variados tipos de violência, como violências física e sexual, emocional e psicológica, isolamento social, violência econômica, violência doméstica, acidentes traumáticos, óbitos e/ou tratamento do trauma de agressores e vítimas, entre outros ${ }^{(1)}$.

A especialidade forense abrange diversas áreas de competência, incluindo atenção as vítimas de violência, desastres de massa, sistema prisional e psiquiátrico, entre outros). Dentre estes, a violência tornou-se um grande problema social e tem crescido alarmando a sociedade, fazendo com que os países gerassem políticas governamentais, além do aperfeiçoamento da ciência forense onde trabalha junto ao público em casos de violência e a justiça, contribuindo com entregas de vestígios ${ }^{(2)}$.

Os vestígios são vitais para o sucesso de uma investigação, pois podem contribuir para a descoberta de uma solução que conduza à resolução dos processos-crime. A investigação criminal procura a descoberta da verdade, com vista à perseguição penal dos agentes do crime, e o estudo dos vestígios é decisivo para provar a culpa ou a inocência de um determinado suspeito ${ }^{(3)}$.

"No campo de ação de uma investigação de agressão sexual, é fundamental pesquisar os vestígios deixados no corpo ou nas peças de vestuário da vítima”"(4).

O crime violento e os traumas associados são entidades que envolvem a atuação de vários profissionais. Os Enfermeiros como profissionais de saúde têm como principal objetivo a assistência à vítima promovendo cuidados de saúde. Apesar desta vital prioridade, é também importante que estes procedam à identificação apropriada, segurança e preservação dos vestígios (biológicos e não biológicos) encontrados no corpo da vítima/ agressor ou no local, que mais tarde poderão vir a ser alvo de prova pericial em Tribunal com relevância Médico-Legal(5).

Levando em consideração dos desdobramentos relatados o estudo justificou-se pela notória importância em aprofundar o conhecimento sobre a temática de forma a tentar viabilizar a preservação de vestígios em caso de violência sexual de forma a facilitar a investigação do ocorrido. A ideia da temática surgiu a partir do interesse de saber o que a literatura traz acerca da função do enfermeiro forense na preservação de vestígios em casos de violência sexual.

Considerando os aspectos descritos, os quais apontam que o desaparecimento de vestígios inviabiliza o processo investigativo, este estudo objetivou descrever a atuação do enfermeiro na preservação de vestígios forenses em casos de violência sexual.

\section{MÉTODOS}

Trata-se de uma revisão integrativa orientada pelas etapas propostas por Mendes, Silveira e Galvão(19): 1. escolha e definição do tema (elaboração da questão de pesquisa); 2. estabelecimento dos critérios de inclusão e exclusão de estudos; 3. busca na literatura (amostragem); 4. critérios para categorização dos estudos (coleta de dados); 5. avaliação dos estudos incluídos nos resultados; 6 . discussão do resultado e apresentação da revisão integrativa. 
Dessa forma, buscou responder a seguinte pergunta de pesquisa: "Como atua o enfermeiro na preservação de vestígios forenses em casos de violência sexual? Esta questão foi definida conforme o acrônimo P.I.Co ( $\mathrm{P}=$ Participante, $\mathrm{I}=$ Fenômeno de interesse, $\mathrm{Co}=$ Contexto do Estudo ${ }^{(20)}$. A análise das evidências da literatura científica sobre a temática foi realizada durante o mês de setembro de 2020 buscaram-se os estudos de forma pareada, no Decs: Cuidados de Enfermagem, Delitos Sexuais, Enfermagem Forense, Saúde da Mulher, Serviços de Saúde da Mulher e no MeSh: Nursing Care, Sex Offenses, Forensic Nursing, Women's Health e Women's Health Services, utilizando-se do operador boleando AND para o cruzamento nas bases de dados. O acesso aos estudos deu-se através do Portal de Periódicos da Coordenação de Aperfeiçoamento de Pessoal de Nível Superior (CAPES), selecionaram-se as bases de dados conforme o escopo de abrangência e a representatividade, as quais foram: Medical Literatura Latino americana e do Caribe em Ciências da Saúde (LILACS), Base de Dados em Enfermagem (BDENF) e Scientific Electronic Library Online.

Para realizar a filtragem dos estudos, conforme a ênfase do objeto investigado, empregaram-se os critérios de inclusão: artigos de pesquisa qualitativas e/ou quantitativas que abordassem a atuação do enfermeiro na preservação de vestígios forenses em casos de violência sexual, publicados nos idioma português, inglês ou espanhol, entre os de 2015 á 2020, disponíveis on-line, na íntegra e gratuitamente nas bases de dados. Ressalta-se que foram incluídos estudos com vários tipos de delineamentos e evidências científicas, devido à característica da questão norteadora, que não se relaciona à eficácia de uma intervenção, a qual, necessariamente, remeteria a estudos experimentais com níveis de evidência forte, mas, sim, à abrangência do conhecimento produzido acerca de determinada temática. Aos critérios de exclusão: Os cri-
Quadro 1- Seleção dos artigos de pesquisa nas bases de dados LILACS, Scielo e BDENF de acordo com os critérios de inclusão estabelecidos.

\begin{tabular}{|l|c|}
\hline & TOTAL \\
\hline Produção encontrada & 45 \\
\hline Não está disponivel online & 15 \\
\hline Repetido & 10 \\
\hline Não aborda a temática & 14 \\
\hline Total selecionado & 06 \\
\hline Fonte: Elaborado pelas autoras (2020). & \\
\hline
\end{tabular}

térios de exclusão estabelecidos foram: artigos que abordassem outros tipos de violência, público-alvo da pesquisa que não fossem mulheres violentadas, reportagens, estudos de desenvolvimento de tecnologias, artigos de revisão, teses, dissertações, estudos duplicados e editoriais.

A seleção dos artigos ocorreu em duas etapas. Na primeira etapa foi realizada a leitura minuciosa dos títulos e resumos de 45 estudos encontrados nas bases de dados supracitadas. Após a aplicação os critérios de inclusão e efetuar a exclusão de 10 artigos repetidos e 15 que não estão disponíveis na íntegra restaram 20 artigos. $\mathrm{Na}$ segunda etapa realizou-se a leitura na íntegra dos artigos e exclusão de 14 artigos que não abordam a temática. A amostra final foi composta por 6 estudos. A seleção foi apresentada no (Quadro 1).

Para análise dos estudos, aplicou-se instrumento de elaboração própria, com inclusão dos itens: autores/país/ano; tipo de estudo e nível de evidência; objetivos e resultados.

\section{RESULTADOS}

$\mathrm{Na}$ presente revisão integrativa, analisou-se 06 artigos que atenderam aos critérios de inclusão previamente estabelecidos e, a seguir, apresentou-se um panorama geral dos artigos avaliados.

O quadro 2 nos mostra estudo que foram publicados em revistas variadas contando com 6 periódicos. Com relação ao tipo de revista científica na qual foram

Quadro 2 - Caracterização dos estudos quanto aos autores, ano, tipo de estudo, objetivos e principais resultados.

\begin{tabular}{|l|c|c|c|}
\hline AUTORES/ANo & $\begin{array}{c}\text { TIPO DE } \\
\text { ESTUDO }\end{array}$ & OBJETIVOS & RESULTADOS \\
\hline $\begin{array}{l}\text { Rocha, Rodrigues, Paula, } \\
\text { Araújo, Gomes, Souza, Sa- } \\
\text { chett e Carvalho (2020) }\end{array}$ & Qualitativo & $\begin{array}{c}\text { Refletir a atuação do enfermeiro fo- } \\
\text { rense com a equipe multidisciplinar na } \\
\text { preservação dos vestígios criminais } \\
\text { durante o atendimento hospitalar de } \\
\text { urgência e emergência }\end{array}$ & $\begin{array}{c}\text { É possível perceber o quanto se faz necessário } \\
\text { os enfermeiros na atuação dos Serviços de } \\
\text { Urgência e/ou Emergência, pois os mesmos } \\
\text { terão contato direto com as vítimas que em sua } \\
\text { maioria estarão cercadas de traumas }\end{array}$ \\
\hline $\begin{array}{l}\text { Santos, Eloi, Santos, } \\
\text { Santos e Silva (2019) }\end{array}$ & $\begin{array}{c}\text { Analítico, } \\
\text { descritivo e } \\
\text { quantitativo. }\end{array}$ & $\begin{array}{c}\text { Analisar o grau de conhecimento e } \\
\text { executabiliade dos profissionais de } \\
\text { saúde na preservação dos vestígios } \\
\text { forenses no atendimento a pacientes } \\
\text { vítimas de violência sexual no hospital } \\
\text { de trauma de Aracaju }\end{array}$ & $\begin{array}{c}\text { Os resultados mostraram que quanto maior } \\
\text { o nível de conhecimento, maior a execução de } \\
\text { procedimentos relacionados à documentação, } \\
\text { preservação e coleta de vestígio }\end{array}$ \\
\hline
\end{tabular}




\begin{tabular}{|c|c|c|c|}
\hline Gomes $(2018)^{16}$ & Qualitativo & $\begin{array}{l}\text { Apresentar as diversas situações } \\
\text { criminógenas que ocorrem no con- } \\
\text { texto pré- hospitalar e apresentar os } \\
\text { respectivos vestígios que devem ser } \\
\text { preservados. }\end{array}$ & $\begin{array}{l}\text { Deve ser deixado no local o material descartá- } \\
\text { vel, nomeadamente as luvas, que devem ser } \\
\text { colocadas em sacos de papel, e estes devem ser } \\
\text { selados e entregues à autoridade. }\end{array}$ \\
\hline $\begin{array}{l}\text { Camilo, Dantas, Musse, } \\
\text { Silva e Assis }(2017)^{11}\end{array}$ & Qualitativo & $\begin{array}{l}\text { Conhecer o papel do enfermeiro na } \\
\text { preservação de vestígios forenses nos } \\
\text { serviços de urgência e emergência }\end{array}$ & $\begin{array}{l}\text { Muito tem dificuldades durante os procedi- } \\
\text { mentos para coleta e conservação dos vestígios } \\
\text { sendo a inexistência de protocolos de orientação }\end{array}$ \\
\hline $\begin{array}{l}\text { Santos, Lucas, Ferro, Ma- } \\
\text { tos e Rosa }(2017)^{15}\end{array}$ & $\begin{array}{l}\text { Analítico, } \\
\text { descritivo e } \\
\text { quantitativo. }\end{array}$ & $\begin{array}{l}\text { Destacar a atuação e as compe- } \\
\text { tências do enfermeiro forense com } \\
\text { enfoque na preservação de vestígios } \\
\text { nos serviços de urgência e emer- } \\
\text { gência, mostrando a importância do } \\
\text { seu papel, assim como aprofundar o } \\
\text { conhecimento nessa área }\end{array}$ & $\begin{array}{c}\text { É possível perceber a necessidade do enfermei- } \\
\text { ro forense nas diferentes categorias de atuação, } \\
\text { visto que ele deve estar preparado para prestar } \\
\text { uma assistência qualificada e especializada a } \\
\text { vítimas e aos agressores. }\end{array}$ \\
\hline $\begin{array}{l}\text { Souza, Martins e Silva. } \\
(2017)^{18}\end{array}$ & $\begin{array}{l}\text { Analítico, } \\
\text { descritivo e } \\
\text { Quantitativo. }\end{array}$ & $\begin{array}{l}\text { Investigar a preservação de vestígios } \\
\text { pelo enfermeiro durante o atendi- } \\
\text { mento de mulheres vítimas de violên- } \\
\text { cia sexual em um serviço de urgência } \\
\text { e emergência do estado de Sergipe. }\end{array}$ & $\begin{array}{l}\text { Os resultados evidenciam que os enfermeiros } \\
\text { atuantes no serviço de emergência no local do } \\
\text { estudo, embora considere importante a preser- } \\
\text { vação e coleta de vestígios frente ao atendi- } \\
\text { mento à mulher vítima de violência sexual, não } \\
\text { se sentem preparados técnicos e cientificamen- } \\
\text { te para desempenhá-los. }\end{array}$ \\
\hline Fonte: Elaborado pelas autoras (2020). & & & \\
\hline
\end{tabular}

publicados os artigos selecionados, percebeu-se que todos estavam enquadrados em revistas de saúde, um fator favorável para a disseminação do conhecimento científico produzido pela saúde, haja vista que o público-alvo que procura o assunto em questão também será beneficiado com as atualidades sobre o tema.

Dos estudos encontrados (4) eram de abordagem qualitativa e (2) de abordagem quantitativa. $\mathrm{O}$ público alvo dos artigos era variado.

\section{DISCUSSÃO}

Os profissionais da área de saúde são os primeiros a ter contato com os agredidos, principalmente o corpo de enfermagem do hospital. As ciências forenses geralmente começam na cena do crime. Caso os vestígios não sejam reconhecidos e cuidadosamente recolhidos, preservados e registrados, pouco ou nada poderá ser efetuado a nível laboratorial, de forma a contornar este problema ${ }^{(10)}$.

O enfermeiro (a) é o profissional que se encontra na linha de frente em relação ao cuidado aos pacientes e consequentemente o(a) primeiro(a) a receber as vítimas de

\section{Os profissionais}

da área de saúde

são os primeiros

a ter contato com

os agredidos,

principalmente

o corpo de

enfermagem do

hospital. As ciências

forenses geralmente

começam na cena do

crime. violência quando estas chegam aos serviços de saúde; a atuação no enfrentamento a violência é ampla e complexa abrangendo desde a identificação de sinais físicos e comportamentais, bem como ações educativas voltadas a família e comunidade, além da notificação compulsória. Entretanto, nem sempre esses(as) profissionais estão aptos(as) a lidar com esse cenário, ou seja, os(as) enfermeiros(as) não são capacitados para lidar com vítimas decorrentes de situação de violência ${ }^{(11-21)}$.

Embora a prioridade seja o acolhimento, a avaliação das lesões e a manutenção da vida, são nesses ambientes que estão as primeiras oportunidades para coleta de vestígios. Nesse cenário, os enfermeiros são privilegiados para identificação e conservação, entretanto poucos estudos abordam sua participação para a descoberta de evidências. Muitos têm dificuldades durante os procedimentos para a coleta e conservação dos vestígios sendo a inexistência de protocolos de orientação ajustados a realidade de cada país um dos aspectos contribuintes para isso ${ }^{(12)}$.

Estudos realizados acerca do conhecimento e executabilidade dos profissionais de saúde na preservação dos vestí- 
gios forenses a vítimas de violência sexual mostraram que quanto maior o nível de conhecimento, maior a execução de procedimentos relacionados a documentação, preservação e coleta de vestígios. Entretanto, a maioria dos sujeitos não se sente preparado para realizar a preservação e coleta de vestígios e que a maior parte dos procedimentos listados não são executados pelos profissionais, embora sejam conhecidos. Verificou-se também que as ações mais executadas são aquelas relacionados a documentação, divergindo dos procedimentos referentes a coleta de vestígios que apresentou menor execução ${ }^{(13)}$.

$\mathrm{O}$ enfermeiro forense tem um grande papel dentro no cenário de violência sexual, contribuindo desde o reconhecimento, abordagem e auxílio em tribunais. É necessário, portanto que esteja atento a quaisquer indícios de violência, para que possa tomar condutas sem suposiçóes errôneas, sempre preservando a vítima e o processo que a mesma passará após o reconhecimento da violência sexual sofrida. A atuação do enfermeiro forense permite que o profissional possa coletar materiais para exames, realizar exames físicos para detecção e tratamento da violência e trauma, e ainda oferecer apoio emocional as vítimas de estupro e seus familiares. Participa na investigação da causa da morte da vítima, sendo um auxílio aos peritos e podendo se fazer testemunha em tribunais, se necessário, atende sobreviventes de todo e qualquer tipo de violência e negligência, incluindo tráfico de humanos, erros médicos, entre outras situações ${ }^{(1)}$.

Nos países em que a enfermagem forense possui legislação que garante a cadeia de custódia e atuam na coleta de vestígios forenses, em situações de violência sexual, nas quais comumente acontece contato físico entre a vítima e o agressor, há a troca de diversos materiais além de fluidos corporais, assim como fragmentos de pele, fibras, vidros e terra. Para garantir a integridade de alguns desses vestígios deve-se atentar ao recolhimento minucioso das roupas separadamente. Sua retirada deve ser feita cautelosamente, quando for necessário, cortá-las pelas costuras, afastan- do e preservando a área de lesão. Outro aspecto importante é não deixar entrar em contato com o chão, evitando a contaminação cruzada. É recomendável o uso de um lençol limpo no chão e sobre ele uma folha de papel. Os sapatos também preci-

\section{O enfermeiro forense} tem um grande papel dentro no cenário de violência sexual, contribuindo desde o reconhecimento, abordagem e auxílio em tribunais. É necessário, portanto que esteja atento a quaisquer indícios de violência, para que possa tomar condutas sem suposiçôes

$$
\text { errôneas, sempre }
$$

preservando a vítima

e o processo que a mesma passará após o reconhecimento da violência sexual sofrida. sam ser recolhidos em pacote individual, pois poderão conter amostras de solo ${ }^{(14)}$.

Quando atuante nos cenários de crime, o enfermeiro forense deve aproximar-se com cuidado, entrar e sair pelo mesmo acesso e evitar a circulação pelo cenário, de forma a minimizar o risco de contaminação e alterações do local. É imprescindível o registro de informações pertinentes e a utilização de técnicas especifica buscando preservar o local e não atrapalhar o processo de avaliação da perícia ${ }^{(13,14)}$. Nos casos de peças de roupas que contenham informações relevantes, as mesmas devem ser empacotados em sacos de papel, frascos de vidro ou de plástico, e dobradas para precaução colocar um folha de papel entre as partes, no caso da peça de roupa estar molhada o enfermeiro deve secar antes de empacotar, caso as mãos da vítima contenha vestígios, se possível as mãos devem ser contidas em saco de papel selados até o cotovelo para análise ${ }^{(15)}$.

Sabe-se que o conhecimento é a base de todo processo, entretanto, há uma grande dificuldade em relação ao conhecimento prévio e técnicas de coleta e conservação dos vestígios forenses pelos enfermeiros dos serviços de urgência e emergência $(16,17)$. A escassez de conhecimento científico referente à violência e enfermagem forense levam aos profissionais de saúde encontrar dificuldades na utilização de técnicas específicas para a preservação dos indícios, contribuindo para o comprometimento e a perda de amostras, não otimização do tempo, desgaste físico e emocional da vítima e profissional, não conclusão do caso e perpetuação do agravo. Perante isso, nota-se a necessidade do enfermeiro forense nos diferentes cenários de atuação, visto que é o mais preparado para prestar uma assistência qualificada e especializada a vítimas e agressores. Nota-se ainda a carência da inclusão do assunto nas diretrizes curriculares dos cursos de formaçã̃o ${ }^{(18)}$.

\section{CONCLUSÃO}

Conclui-se que, o enfermeiro forense possui uma gama de responsabilidade perante os sobreviventes de violência se- 
xual, desde a abordagem até o manuseio adequado das provas. No entanto, existe ainda despreparo e falta de treinamento, sendo destacadas como as principais limitações. A qualificação profissional é outro fator relevante, visto que em decorrência de uma abordagem incipiente sobre esse tema nos cursos de graduação, tanto no aspecto teórico como prático, somado a falta de treinamento e protocolos nos serviços. Portanto faz-se necessário a inserção de conteúdos envolvendo a problemática nas disciplinas, assim como a realização de projetos extensionistas e/ou de pesquisas em consonância com os serviços. Observou-se ainda que há carência de artigos sobre a temática. Assim a saúde pública apresenta seu olhar pontual com mudan- ças na qualidade de vida e padrão de saúde.

Espera-se que esse trabalho possa contribuir com discussões mais aprofundadas sobre a temática, pois existe certa carência do papel fundamental da enfermagem em artigos escritos, ressaltando-se a extrema importância de um conhecimento prévio sobre abordagem e conservação dos vestígios em casos de violência sexual.

\section{REFERÊNCIAS}

1. Silva KB, Silva RC. Enfermagem Forense: uma especialidade a conhecer. Cogitare Enfermagem, v. 14, n. 3, p. 564-568,jul/set. Curitiba, Paraná. 2009.

2. Associação Brasileira de Enfermagem Forense. Parecer sobre campo de atuação da Enfermagem Forense Brasileira protocolado no COFEN. Brasília, 2015. Disponivel em: https://www.abeforense.org.br/parecer-sobre-campo-de-atuacao-da-enfermagem-forensebrasileira-protocolado-no-cofen/. Acesso em 20 de set., 2020.

3. Silva G, Soares FG. Preservação da Cadeia de Custódia em vestígios biológicos para fins forenses : caracterização da situação actual e proposta de critérios de recolha e envio de vestígios biológicos. Lisboa : - 130 - Faculdade de Medicina da Universidade de Lisboa, 2006. Tese de Mestrado.

4. Pinheiro, Maria de Fátima Terra (org.). (2008). - CSI Criminal. Porto: Universidade Fernando Pessoa.

5.. Pompeo DA, Rossi L A, Galvão CM. Revisão integrativa: etapa inicial do processo de validação de diagnóstico de enfermagem. Acta. Paul. Enferm.São Paulo, v. 22, n. 4, 2009.

6. Mendes KD, Silveira RCCP, Galvão CM. Revisão integrativa: método de pesquisa para a incorporação de evidências na saúde e na enfermagem integrativa literatura. Texto \& Contexto Enferm, Florianópolis, v. 17, n. 4, p. 758-764, out/dez, 2008.

7. Lakatos EM, Markoni MA. Metodologia do trabalho científico: projetos de pesquisa / pesquisa bibliográfica/ teses de doutorado, dissertações de mestrado, trabalhos de conclusão de curso / Marina de Andrade Marconi, Eva Maria Lakatos. - 8. ed. - São Paulo: Atlas, 2017.

8. Minayo MCS, Deslandes SF, Gomes R. Pesquisa Social: Teoria, método e criatividade. 28. ed. Rio de Janeiro: Vozes, 2009. 108 p.

9. Rocha HN, Rodrigues BA, Paula GVN, Araujo JPA, Gomes TA, Souza ARN, Sachett JAG, Carvalho ES. O enfermeiro e a equipe multidisciplinar na preservação de vestígios forenses no serviço de urgência e emergência. Brazilian Journal of health Review. 2020; v.3, n.2; 2208-2217. doi: 10.34119/bjhrv3n2-073.

10. Martins DC, et al. Violência: Abordagem, atuação e educação em enfermagem. Ciências Biológicas e de Saúde Unit, v.4, n.2, p. 155-168, out. 2017.

11.Camilo LSS, et al. Preservação da cena do crime pelo enfermeiro no serviço de atendimento móvel de urgência: Uma revisão integrativa. Ciências biológicas e de saúde UNIT. 2017. v.4, n.2;
185-202. ISSN 2316-3143.

12. Santos FP, et al. Conhecimento e atuação de profissionais de saúde na preservação de vestígios forenses em pacientes vítimas de violência sexual em hospital de trauma em Aracaju. $2^{\circ}$ Congresso Internacional de Enfermagem - $\mathrm{CIE} / 13^{\circ}$ Jornada de Enfermagem da Unit (JEU) - 6 a 10 maio de 2019.

13. Gonçalves SIF. Vivências dos Enfermeiros na Manutenção de Provas Forenses no Serviço de Emergência" [Dissertação]. Portugal: Universidade do Porto, Portugal; 2011.

14. Libório RPG. Práticas de Enfermagem Forense: Conhecimentos em Estudantes de Enfermagem. Mestrado em Enfermagem Médico-Cirurgica - $2^{\circ}$ ed., maio 2012.

15. Santos MR, et al. Atuação e Competência do Enfermeiro Forense na Preservação de Vestígios no Serviço de Urgência e Emergência. In the construction of society May 9-12, 2017.

16. Gomes AM. Preservação de vestígios no pré-hospitalar: papel das equipas de emergência medica em cenários forenses. Nursing. 2018 [cited 2021 Mar 17]. Avaliable from: https://www.nursing.pt/ preservacao-de-vestigios-no-pre-hospitalar-papel-das-equipas-de-emergencia-medica-em-cenarios-forenses/

17. Gomes A. (2014). Enfermagem forense. Vol.1. Lidel, Lisboa.

18. Souza $A C D$, et al. 0 enfermeiro e a preservação de vestígios nos casos de violência sexual. In the construction of society May 9-12, 2017.

19. Rocha HN, Rodrigues BA, Paula GVN, Araújo JPA, Gomes TA, Souza ARN et al. $O$ enfermeiro e a equipe multidisciplinar na preservação de vestígios forenses no serviço de urgência e emergência. Brazilian Journal of Health Review [internet]. 2020; 3 (2): 2208-17. DOl:10.34119/bjhrv3n2-073

20. Mendes KDS, Silveira RCCP, Galvão CM. Revisão integrativa: método de pesquisa para a incorporação de evidências na saúde e na enfermagem. Texto \& Contexto - Enfermagem [internet]. 2008; 17 (4): 758-764. DOl: https://doi.org/10.1590/S010407072008000400018

21. Araujo da Silva K, Martins de Souza AD, de Souza Leite JC, Neves Nóbrega RJ, Brito de Lima M, Xavier Silva JP. Atenção primária à saúde: percepções de enfermeiros/as frente à violência sexual contra crianças e adolescentes. SaudColetiv (Barueri) [Internet]. $9^{\circ}$ de dezembro de 2020 [citado $14^{\circ}$ de abril de 2021];10(59):422435. Disponivel em: http://revistas.mpmcomunicacao.com.br/index.php/saudecoletiva/article/view/1063 